\title{
Meta
}

Journal des traducteurs

Translators' Journal

\section{Ballard, Michel et Lieven D'Hulst (dir) (1996) : La traduction en \\ France à l'âge classique, Villeneuve D'Ascq, Presses Universitaires du Septentrion, 325 p.}

\section{Marc Charron}

Volume 42, numéro 3, septembre 1997

L’interprétation en langues des signes

URI : https://id.erudit.org/iderudit/002222ar

DOI : https://doi.org/10.7202/002222ar

Aller au sommaire du numéro

Éditeur(s)

Les Presses de l'Université de Montréal

ISSN

0026-0452 (imprimé)

1492-1421 (numérique)

Découvrir la revue

Citer ce compte rendu

Charron, M. (1997). Compte rendu de [Ballard, Michel et Lieven D'Hulst (dir) (1996) : La traduction en France à l'âge classique, Villeneuve D'Ascq, Presses Universitaires du Septentrion, 325 p.] Meta, 42(3), 595-597.

https://doi.org/10.7202/002222ar d'utilisation que vous pouvez consulter en ligne.

https://apropos.erudit.org/fr/usagers/politique-dutilisation/ 
BALLARD, Michel et Lieven D'HULST (dir.) (1996): La traduction en France à l'âge classique, Villeneuve d'Ascq, Presses Universitaires du Septentrion, 325 p.

Cet ouvrage collectif sur l'histoire de la traduction en France aux XVII et XVIII ${ }^{\mathrm{e}}$ siècles, dirigé par Michel Ballard et Lieven D'hulst, vient s'inscrire dans la lignée des ouvrages à caractère historique que ces deux chercheurs ont publiés au cours des dernières années chez le même éditeur (les anciennes Presses Universitaires de Lille). La parution de ce dernier ouvrage souligne une fois de plus l'importance grandissante de l'histoire de la traduction comme champ d'étude (ou sous-discipline) de la traductologie; à vrai dire, l'autonomie grandissante de cette sous-discipline n'est pas sans évoquer celle durement acquise par la traductologie même depuis bientôt une génération. La traduction en France à l'âge classique est le carrefour de seize études traductologiques, la plupart descriptives, où sont présentées les multiples façons dont la traduction a participé à l'histoire culturelle française, à la formation d'une littérature nationale émergente, à la formation du goût littéraire à l'époque classique, ainsi qu'au développement de relations hiérarchiques entre le français et d'autres langues - européennes, dans le cas des articles de Graeber et de van Gorp, et non européennes, dans le cas de l'article de St-Pierre, par exemple. Ainsi, dans la présentation de l'ouvrage, les éditeurs rappellent la permanence de certains lieux communs associés à la traduction à l'époque classique (dont l'ineffable belles infidèles), qui n'aurait réussi selon eux qu'à perpétuer une rhétorique complaisante et à simplifier à outrance les complexes réalités qui ont façonné l'histoire de la traduction aux XVII et XVIII ${ }^{\mathrm{e}}$ siècles.

Parmi les aspects importants à retenir, il faut d'abord noter la place de choix accordée dans cet ouvrage aux traductions effectuées à partir de langues vernaculaires (surtout dans la partie de l'ouvrage intitulée «Traductions et littératures»); il nous semble d'autant plus pertinent de rapporter ici ce détail quand on sait l'espace habituel réservé à la problématique vernaculaire dans l'ensemble des travaux sur la traduction avant le 
$\mathrm{XVIII}^{\mathrm{e}}$ siècle. En deuxième lieu, il faut souligner la proportion sinon significative, du moins réelle et non négligeable, consacrée à la traduction d'ouvrages non littéraires (on n'a qu'à penser à l'article de Kittel et à sa description de la fortune éditoriale de l'almanach de Benjamin Franklin en France; ou encore à l'article de Touitou-Benitah, qui nous fait découvrir les premières traductions françaises du texte liturgique de la Pâque juive de la Haggada).

La division de l'ouvrage en trois parties («Parcours théoriques», «Modalités et situations de la traduction» et «Traductions et littératures») reprend les thèmes tels qu'ils avaient été soumis aux participants d'un colloque tenu en 1993 à l'Université Charlesde-Gaulle-Lille III. La première partie, consacrée à la réflexion spécifiquement théorique, compte cinq interventions : celles de Luce Guillerm, «Les Belles Infidèles, ou l'Auteur respecté (de Claude de Seyssel à Perrot d'Ablancourt)», des éditeurs de l'ouvrage de Michel Ballard, «Gaspard de Tende: théoricien de la traduction», et de Lieven D'hulst «Unité et diversité de la réflexion traductologique en France (1722-1789)», de Daniel Mercier, «La problématique de l'équivalence des langues aux XVII ${ }^{\mathrm{e}}$ et XVIII ${ }^{\mathrm{e}}$ siècles», et de José Lambert, «Le discours implicite sur la traduction dans l'Encyclopédie». Entre autres parce qu'elle reprend le fil évolutif de certains topoï jugés propres à la traduction au $\mathrm{XVII}^{\mathrm{e}}$ siècle, l'intervention la plus stimulante de cette première partie de l'ouvrage est sans conteste celle de Guillerm; c'est à la lecture de l'étude de cette spécialiste de la traduction à la Renaissance que le lecteur prend conscience - peut-être davantage qu'il ne lui est possible de le faire ailleurs - de la nécessité de réévaluer les fondements du discours complaisant sur l'histoire de la traduction à l'époque classique. Plus particulièrement, il se dégage ici une réflexion inédite sur la filiation qui existe entre les discours théoriques sur la pratique de la traduction au XVII ${ }^{\mathrm{e}}$ siècle et ceux qui prévalent en pleine Renaissance française. C'est de façon presque dérangeante mais hautement convaincante que l'auteur précise comment s'établit la continuité discursive entre les deux époques et qu'elle présente la notion fondamentale de métempsychose, qui, comme le précisent Ballard et D'hulst dans leur présentation, se superpose à l'idée de naturalisation.

La deuxième partie, consacrée à ce que les éditeurs nomment ici «modalités et situations de la traduction», réunit des articles qui traitent essentiellement des valeurs et normes institutionnelles ayant modelé l'univers traductionnel et traductologique de l'époque: ce sont ceux de Philippe Caron, «Le Quinte Curce de Vaugelas à l'épreuve : une relecture critique à l'Académie Française», d'Alain Lautel, «La fortune française de six auteurs anglais du XVIII ${ }^{\mathrm{e}}$ siècle fondée sur des appréciations numériques», de Harald Kittel, «From Poor Richard to Le bonhomme Richard», de Paul St-Pierre, «L'Orient traduit en français: l'Autre transposé», de Bernard Chédozeau, «Participation à la messe par ou sans la traduction: l'assistance à la messe», et enfin de Colette Touitou-Benitah, «La première traduction française de la Haggada». Bien entendu, il est largement question, dans la plupart des six articles regroupés sous ce thème des modalités, de la situation discursive contemporaine des phénomènes observés; en fait, tous poursuivent un but descriptif commun, soit celui de montrer en quoi la traduction a pu contribuer à la diffusion du discours social qui domine en France à l'âge classique. Le seul aspect qui peut agacer est la surabondance, notamment dans l'article de Lautel, de données et de tableaux statistiques venant littéralement illustrer les résultats de la recherche (et qu'on souhaiterait plutôt voir présentés en annexe).

La troisième et dernière partie, consacrée au thème plutôt vague des «traductions et littératures», est sans doute la plus conventionnelle de l'ouvrage (mais pas pour autant la moins intéressante), en ce qu'elle présente au lecteur des études plus typiques et plus caractéristiques de ce qui se fait habituellement, pour ainsi dire, en histoire de la littérature (comparée ou non); on trouve ici les interventions de Guiomar Hautcoeur, «Les 
Nouvelles Tragi-Comiques de Scarron et la critique du roman», d'Amelia Sanz, «Déplacements sémantiques dans les traductions d'Anne de la Roche-Guilhem», du regretté André Lefevere, «The Extract: Literary Guerilla as Literary Interchange», d'Hendrik van Gorp, «Traductions, versions et extraits dans la Nouvelle Bibliothèque des Romans et la Bibliothèque Britannique (1796-1802)» et de Wilhelm Graeber, «Le charme des fruits défendus : les traductions de l'anglais et la dissolution de l'idéal classique». L'aspect particulièrement intéressant qui est développé dans cette dernière partie de l'ouvrage concerne la question de la traduction de genres littéraires spécifiques (c'est-à-dire qui y sont exposées, non pas tant des problématiques d'ordre général sur la traduction de la poésie, de la prose ou du théâtre, mais des analyses très détaillées sur la traduction de particularités littéraires comme, par exemple, celles de l'extrait (théâtral chez Lefevere et romanesque chez van Gorp) ou de la nouvelle (cf. l'article de Hautcoeur sur le comique comme fonction littéraire populaire au XVII siècle).

De façon plus globale, La traduction en France à l'âge classique apporte une contribution imposante à une «historiographie des théories de la traduction» à bâtir, telle que la disait nécessaire Lieven D'hulst dans un article paru en 1995 dans la revue $T T R^{1}$. (On devinera que les éditeurs du présent volume se réclament d'emblée de cet article et du projet où y avaient été tracées certaines pistes exploratoires.) De ce nouvel effort commun se dégagent les limites temporelles, historiques, mais avant tout idéologiques de ce qu'il est convenu d'appeler l'idéal classique en traduction. Ces limites sont clairement revendiquées, surtout si l'on considère que le premier article, celui de Guillerm, aborde la question de la formation de l'idéal classique; et aussi que le dernier article, celui de Graeber, clôt sur ce que le chercheur allemand appelle la dissolution de ce même idéal. En cela, La Traduction en France à l'âge classique trace un tableau diversifié et assez complet de la place de la traduction au sein des différents modes d'écriture de l'époque.

Assez étrangement, le nom de Zuber qui revient dans bon nombre d'articles nous fait saisir que, tout bien considéré, rares sont les chercheurs en traductologie qui se sont véritablement intéressés depuis la fin des années soixante à la composante historique de la traduction. Cette résurgence spontanée de l'intérêt pour l'histoire de la traduction, auquel contribue en grande partie le présent ouvrage, peut difficilement ne pas être mis en parallèle avec celle qui s'est manifestée depuis une dizaine d'années déjà en littérature, et où il est particulièrment question de réexaminer l'histoire et les façons dont elle a été écrite et présentée au cours des siècles par l'institution littéraire. Le projet de réécriture de l'histoire de la traduction équivaut ici à mettre en relief le rôle formateur, novateur (voire subversif, dans le cas de l'article de Chédozeau, qui raconte comment les laïcs participent au déroulement de la messe «par ou sans la traduction») de la traduction et, conséquemment, à s'interroger sur le besoin urgent de revoir et de réévaluer les ouvrages historiques portant sur la traduction jusqu'ici, afin de déterminer quelles sont les contraintes sociales, institutionnelles et éditoriales propres aux différentes entreprises traductionnelles de l'âge classique français.

En conclusion, il n'y a qu'à souhaiter que d'autres colloques (et leurs actes) portant sur des thèmes similaires verront le jour au cours des prochaines années et que l'appel lancé par D'hulst pour une historiographie des théories de la traduction sera entendu; ainsi les études historiques comme branche autonome des études sur la traduction cesseront tout bonnement d'être perçues comme le produit d'une rhétorique complaisante.

MARC CHARRON Université de Montréal, Montréal, Canada

Note

1. Lieven D'HULST (1995) : «Pour une historiographie des théories de la traduction. Questions de méthode», $T T R, 8 ; 1$, pp. 13-33. 